\title{
Multiply superharmonic functions outside negligible sets
}

\author{
Mohammad Alakhrass ${ }^{1}$
}

Received: 17 March 2015 / Accepted: 8 July 2015 / Published online: 19 July 2015

(C) Fondazione Annali di Matematica Pura ed Applicata and Springer-Verlag Berlin Heidelberg 2015

\begin{abstract}
Let $\Omega_{j} \subset \mathbf{R}^{n_{j}}(j=1,2)$ be an open connected set such that if $n_{j}=2$, it is assumed that $\Omega_{j}$ has a Green function. Let $u$ be a Borel function defined on $\Omega_{1} \times \Omega_{2}$ such that $u$ is locally bounded below and $u \neq \equiv \infty$. Let $\hat{u}$ be the lower semicontinuous regularization of $u$. Let $\mu_{x}^{\omega}$ denote the harmonic measure of $\omega$ at $x$ if $x \in \omega$ and $\mu_{x}^{\omega}=\delta_{x}$ if $x \notin \omega$. Our main result is that $u$ satisfies the inequality
\end{abstract}

$$
u(x, y) \geq \int u d\left(\mu_{x}^{\omega_{1}} \otimes \mu_{y}^{\omega_{2}}\right), \quad \forall(x, y) \in \Omega_{1} \times \Omega_{2}
$$

where $\omega_{1}$ and $\omega_{2}$ are relatively compact open sets with $(x, y) \in \omega_{1} \times \omega_{2} \subset \Omega_{1} \times \Omega_{2}$ if and only if $\hat{u}$ is a multiply superharmonic function and $u=\hat{u}$ outside of a negligible set. This easily implies the extension of Cartan-Brelot convergence theorem on the infimum of a uniformly locally lower bounded family of multiply superharmonic functions on $\Omega_{1} \times \Omega_{2}$.

Keywords Multiply superharmonic functions · Negligible set · Harmonic measure · Cartan-Brelot convergence theorem

Mathematics Subject Classification Primary 31B05; Secondary 31B15 · 31D05 · 32U20

\section{Introduction}

Let $\Omega$ be a connected open subset of $\mathbb{R}^{n}$ for $n \geq 2$; suppose further that $\Omega$ has a Green function defined on it in case $n=2$.

It is well known that a function $u: \Omega \longrightarrow(-\infty, \infty]$ is hyperharmonic if $u$ never takes the value $-\infty$ and

Mohammad Alakhrass

mohammad.alakhrass@mail.mcgill.ca

1 Mathematics Department, College of Science, University of Sharjah,

P.O. Box 27272, Sharjah, UAE 
(a) $u$ is lower semicontinuous on $\Omega$ and

(b) for each $x \in \Omega$ and each regular open set $\omega$ containing $x$,

$$
u(x) \geq \int u d \mu_{x}^{\omega},
$$

where $\mu_{x}^{\omega}$ is the harmonic measure of the regular open set $\omega$ at $x$. A hyperharmonic function $u$ on $\Omega$ is called a superharmonic function if $u \not \equiv \infty$.

A subset $P$ of $\Omega$ is polar (has outer capacity equal zero) if and only if there exists a superharmonic function $u$, on $\Omega$, such that $u=\infty$ on $P$. A Borel probability and compactly supported measure $\mu$ on $\Omega$ is called Jensen measure at $x \in \Omega$ if every superharmonic function $u$ satisfies $u(x) \geq \int u d \mu$.

It is natural to ask what class of functions can be obtained if the continuity condition (a) in the above characterization, of hyperharmonic functions, is relaxed. Concerning this question, in [5], Cole and Ransford proved the following result:

Theorem 1.1 Let $\Omega$ be an open subset of $\mathbb{R}^{n}, n \geq 2$. Let $u: \Omega \longrightarrow(-\infty, \infty]$ be a function which is locally bounded below on $\Omega$, and let $\hat{u}$ be its lower semicontinuous regularization. Suppose further that $\{x \in \Omega: u(x)>t\}$ is analytic for each $t \in \mathbb{R}$. Then, for each $x \in \Omega$ and each Jensen measure $\mu$ for $x$,

$$
u(x) \geq \int u d \mu
$$

if and only if $\hat{u}$ is hyperharmonic and $\hat{u}=u$ outside a polar set.

The authors in a later paper [6] proved implicitly that in the above theorem, it is enough to consider the harmonic measures. The authors, in [5], called the function $u$ which satisfies the above theorem quasi superharmonic function. Extension of Cole and Ransford result to the general axiomatic setup of Brelot spaces has been given. See [2] and [3].

In the sequel, let $\Omega_{1}, \Omega_{2}$ be two Euclidean domains as above (not necessarily of the same dimension). Let $U$ be an open subset of $\Omega_{1} \times \Omega_{2}$. A function $u: U \rightarrow(-\infty, \infty]$ is said to be multiply superharmonic if $u$ is lower semicontinuous, $u>\infty, u \neq \equiv$ in any connected component of $U$, and for any fixed variable, $u$ is hyperharmonic in the other variable. See [7] and [9].

For an open set $\omega \subset \Omega$, the measure $\mu_{x}^{\omega}$ is defined as the harmonic measure of $\omega$ at $x$ if $x \in \omega$ and it is defined to be $\delta_{x}$ if $x$ does not lie in $\omega$. Let $(x, y) \in \Omega_{1} \times \Omega_{2}$. The set $J_{(x, y)}(M S)$ is defined to be the set of all Borel probability and compactly supported measures $\mu$ on $\Omega_{1} \times \Omega_{2}$ such that every multiply superharmonic function $u$ satisfies $u(x, y) \geq \int u d \mu$. It is clear that $\left\{\mu_{x}^{\omega_{1}} \otimes \mu_{y}^{\omega_{2}},:(x, y) \in \omega_{1} \times \omega_{2} \subset \subset \Omega_{1} \times \Omega_{2}\right\} \subset J_{(x, y)}(M S)$. In fact, the measures $\mu_{x}^{\omega_{1}} \otimes \mu_{y}^{\omega_{2}}$ are extreme element in $J_{(x, y)}(M S)$. See [1].

A subset $E$ of $\Omega_{1} \times \Omega_{2}$ is said to be negligible in $\Omega_{1} \times \Omega_{2}$ if there exist polar sets $P_{1}, P_{2}$ in $\Omega_{1}, \Omega_{2}$, respectively, such that $\forall x \in \Omega_{1} \backslash P_{1}$ the section $E_{x}\left(\Omega_{2}\right)=\left\{y \in \Omega_{2}:(x, y) \in E\right\}$ is polar in $\Omega_{2}$, and $\forall y \in \Omega_{2} \backslash P_{2}$ the section $E_{y}\left(\Omega_{1}\right)=\left\{x \in \Omega_{1}:(x, y) \in E\right\}$ is polar in $\Omega_{1}$. [10], [12].

In this article, we extend the above results of Cole and Ransford to a class of Borel functions $u$ defined on $\Omega_{1} \times \Omega_{2}$ satisfying certain super mean value inequalities. More precisely, we investigate the nature of the class of Borel functions $u$, defined on $\Omega_{1} \times \Omega_{2}$ such that $u$ is locally bounded below and for each point $(x, y) \in \Omega_{1} \times \Omega_{2}$

$$
u(x, y) \geq \int u d\left(\mu_{x}^{\omega_{1}} \otimes \mu_{y}^{\omega_{2}}\right),
$$

where $\omega_{1}$ and $\omega_{2}$ are relatively compact open subsets of $\Omega_{1}, \Omega_{2}$, respectively. 
The main result of this article, Theorem 2.4, states: Let $u$ be a Borel function defined on $\Omega_{1} \times \Omega_{2}$ such that $u$ is locally bounded below. Let $\hat{u}$ be the lower semicontinuous regularization of $u$. Then, $u$ satisfies (1) for every pair of domains $\omega_{j} \subset \subset \Omega_{j}(j=1,2)$ if and only if $\hat{u}$ is multiply superharmonic on $\Omega_{1} \times \Omega_{2}$ and $u=\hat{u}$ outside a negligible set.

A product of only two open sets, $\Omega_{1} \times \Omega_{2}$, is considered for simplicity, but the work carries over without a significant change in the case of a finite product of such open sets. Also, even the proof of Theorem 2.4 is valid under the weaker assumption that the set $\{x: u(x)>t\}$ is analytic for all $t \in \mathbb{R}$, we think it is more natural, in the product spaces, to assume that the function $u$ is a Borel function. The strategy in the proofs is similar to that used by Singman to extend the Cartan-Brelot convergence theorem to a finite product of Brelot spaces. See [12].

Finally, we remark that the method of proof of the results in this article does not make particular use of the structure of Euclidean spaces. The results are valid in the more general setting of the product of Brelot harmonic spaces. The spaces we include are Brelot spaces where the sheaf of harmonic functions contains 1 , has a positive potential, and verifies Axiom D.

\section{Proof of main result}

The following theorem will be used in the proof of Theorem 2.4. The proof can be found in [2], [3].

Theorem 2.1 Let $\Omega$ be a connected open subset of $\mathbb{R}^{n}, n \geq 2$. Let $u$ be an extended realvalued Borel function, defined on $\Omega$ such that $u$ is locally bounded below. Then, the following are equivalent:

1. For each $x \in \Omega$, and for each relatively compact open set $\omega$ containing $x$, $u$ satisfies the following inequality

$$
u(x) \geq \int u d \mu_{x}^{\omega}
$$

where $\mu_{x}^{\omega}$ is the harmonic measure of $\omega$ at $x$.

2. $\hat{u}$ is a hyperharmonic function and $u=\hat{u}$ outside of a polar set.

Let $u$ be an extended real-valued function on $U \subset \Omega_{1} \times \Omega_{2}$ such that $u$ is locally lower bounded. Then, $u$ is called a nearly multiply superharmonic function if for all regular domains $\omega, \sigma$ such that $\omega \times \sigma \subset \bar{\omega} \times \bar{\sigma} \subset U$ and $\forall(x, y) \in \omega \times \sigma$ we have the inequality: $u(x, y) \geq \bar{\int} u d\left(\mu_{x}^{\omega} \otimes \mu_{y}^{\sigma}\right)$. Recall that $\bar{\int} u d\left(\mu_{x}^{\omega} \otimes \mu_{y}^{\sigma}\right)=\inf _{\psi} \int \psi d\left(\mu_{x}^{\omega} \otimes \mu_{y}^{\sigma}\right)$, where the infimum is taken over all lower semicontinuous functions $\psi$ such that $\psi \geq u$. See [8].

We introduce the following notation. If $u$ is an extended real-valued function on an open set $U \subset \Omega_{1} \times \Omega_{2}, \hat{u}, \hat{u}^{1}$, and $\hat{u}^{2}$ are defined by

$$
\begin{aligned}
\hat{u}\left(x_{0}, y_{0}\right) & =\liminf _{U \ni(x, y) \rightarrow\left(x_{0}, y_{0}\right)} u(x, y), \\
\hat{u}^{1}\left(x_{0}, y_{0}\right) & =\liminf _{U \ni\left(x, y_{0}\right) \rightarrow\left(x_{0}, y_{0}\right)} u\left(x, y_{0}\right), \\
\hat{u}^{2}\left(x_{0}, y_{0}\right) & =\liminf _{U \ni\left(x_{0}, y\right) \rightarrow\left(x_{0}, y_{0}\right)} u\left(x_{0}, y\right) .
\end{aligned}
$$

The following result is very important in our proofs. See [8]. 
Theorem 2.2 Let $U$ be an open subset of $\Omega_{1} \times \Omega_{2}$. Let $u$ be a nearly multiply superharmonic function on $U$, and $(x, y) \in U$. Let $\omega_{1}^{n}$ be a sequence of regular domains in $\Omega_{1}$ such that $\bigcap_{n} \omega_{1}^{n}=\{x\}$, and let $\omega_{2}^{m}$ be a sequence of regular domains in $\Omega_{2}$ such that $\bigcap_{m} \omega_{2}^{m}=\{y\}$. Then, $u$ satisfies the following equations:

$$
\begin{aligned}
\hat{u}(x, y) & =\sup _{n, m} \int u d\left(\mu_{x}^{\omega_{1}^{n}} \times \mu_{y}^{\omega_{2}^{m}}\right), \\
\hat{u}^{1}(x, y) & =\sup _{n} \int u(., y) d \mu_{x}^{\omega_{1}^{n}}, \\
\hat{u}^{2}(x, y) & =\sup _{n} \int u(x, .) d \mu_{y}^{\omega_{2}^{n}} .
\end{aligned}
$$

Moreover, each sup in the above equations is a limit of an increasing sequence.

Now, we introduce the definition of quasi multiply superharmonic function which is a special case of nearly superharmonic functions and an analog to the Cole and Ransford definition of quasi superharmonic functions.

Definition 2.1 Let $U$ be a connected open subset of $\Omega_{1} \times \Omega_{2}$. Let $u$ be a Borel function, defined on $U$, such that $u \not \equiv \infty$ and $u$ is locally lower bounded. $u$ is called a quasi multiply superharmonic function on $U$ if for each $(x, y) \in U, u$ satisfies the following inequality:

$$
u(x, y) \geq \int u d\left(\mu_{x}^{\omega} \otimes \mu_{y}^{\sigma}\right),
$$

for all relatively compact open sets $\omega, \sigma$ such that $\omega \times \sigma \subset \bar{\omega} \times \bar{\sigma} \subset U$. Here, $\mu_{x}^{\omega}$ is the harmonic measure of $\omega$ at $x$ if $x \in \omega$ and $\mu_{x}^{\omega}=\delta_{x}$ if $x \notin \omega$.

It can be seen easily from the definition that the limit of an increasing sequence of quasi multiply superharmonic functions is a quasi multiply superharmonic function. Also, if $u$ is a quasi multiply superharmonic function, then for any fixed variable, $u$ is quasi superharmonic in the other variable.

It is clear that the function $\hat{u}$ is measurable with respect to the product of harmonic measures because it is lower semicontinuous. Now, we show that $\hat{u}^{1}$ and $\hat{u}^{2}$ are also measurable when $u$ is a quasi multiply superharmonic function. By symmetry, it is enough to show that only one of them is measurable with respect to the product of harmonic measures.

Lemma 2.1 If u is a quasi multiply superharmonic function, then $\hat{u}^{1}$ is a measurable function with respect to the product of harmonic measures.

Proof To show that $\hat{u}^{1}$ is a measurable function, we need to show that the set

$$
A=\left\{(x, y) \in \Omega_{1} \times \Omega_{2}: \hat{u}^{1}(x, y)>a\right\}
$$

is a measurable set for every $a \in \mathbb{R}$. Let $\omega_{n}$ be a countable regular base of $\Omega_{1}$. Then,

$$
A=\bigcup_{n}\left\{(x, y) \in \omega_{n} \times \Omega_{2}: w_{n}(x, y)>a\right\} \text { where } w_{n}(x, y)=\int u(., y) d \mu_{x}^{\omega_{n}} .
$$

The function $w_{n}(x, y)=\int u(., y) d \mu_{x}^{\omega_{n}}$ on $\omega_{n} \times \Omega$ is continuous in the first variable, in fact is harmonic, and is Borel in the second variable (by Fubini's Theorem). Therefore, the result follows by [Theorem 2 [11]].

Now, we prove the first part of our main result, Theorem 2.4. 
Theorem 2.3 Let $u$ be a quasi multiply superharmonic function on $\Omega_{1} \times \Omega_{2}$. If $\hat{u}$ is the lower semicontinuous regularization of $u$, then $\hat{u}$ is multiply superharmonic on $\Omega_{1} \times \Omega_{2}$.

Proof By definition, $\hat{u}$ is lower semicontinuous. $\hat{u}$ never takes the value $-\infty$ because the function $u$ is locally lower bounded. So, we need to show that for any fixed variable, $\hat{u}$ is superharmonic in the other variable. Let $(x, y) \in \Omega_{1} \times \Omega_{2}$. Let $\omega_{1}$ and $\omega_{2}$ be two relatively compact regular domains such that $(x, y) \in \omega_{1} \times \omega_{2} \subset \bar{\omega}_{1} \times \bar{\omega}_{2} \subset \Omega_{1} \times \Omega_{2}$. Since $u$ is a quasi multiply superharmonic function,

$$
u(x, y) \geq \iint u d \mu_{x}^{\omega_{1}} d \mu_{y}^{\omega_{2}} .
$$

This implies that

$$
\hat{u}(x, y) \geq \iint u d \mu_{x}^{\omega_{1}} d \mu_{y}^{\omega_{2}}
$$

since the function $(s, t) \mapsto \iint u d \mu_{s}^{\omega_{1}} d \mu_{t}^{\omega_{2}}$ is continuous.

Using the fact that $u \geq \hat{u}$, we have

$$
\hat{u}(x, y) \geq \iint \hat{u} d \mu_{x}^{\omega_{1}} d \mu_{y}^{\omega_{2}}
$$

Let $\omega_{n}^{1}$ be a sequence of regular domains in $\Omega_{1}$ such that $\bigcap_{n} \omega_{n}^{1}=\{x\}$. Therefore, Eq. (7) implies

$$
\hat{u}(x, y) \geq \iint \hat{u} d \mu_{x}^{\omega_{n}^{1}} d \mu_{y}^{\omega_{2}}
$$

Hence, by Fubini's theorem

$$
\hat{u}(x, y) \geq \liminf _{n} \int\left(\int \hat{u}(s, t) d \mu_{y}^{\omega_{2}}(t)\right) d \mu_{x}^{\omega_{n}^{1}}(s) .
$$

The measure $\mu_{x}^{\omega_{n}^{1}}$ converges weakly to the Dirac measure, and the function $s \rightarrow$ $\int \hat{u}(s, t) d \mu_{y}^{\omega_{2}}(t)$ is lower semicontinuous. Therefore,

$$
\hat{u}(x, y) \geq \int \hat{u}(x, .) d \mu_{y}^{\omega_{2}} .
$$

This proves that $y \rightarrow \hat{u}(x, y)$ is a superharmonic function. Similarly, it can be shown that $x \rightarrow \hat{u}(x, y)$ is superharmonic.

The proof of the following result can be found in [12].

Lemma 2.2 Let $\omega_{1}, \omega_{2}$ be relatively compact domains in $\Omega_{1}, \Omega_{2}$, respectively, and $v$ be a nonnegative locally bounded multiply superharmonic function on an open set containing $\bar{\omega}_{1} \times \bar{\omega}_{1}$. Then, the function

$$
\bar{v}: \omega_{1} \times \omega_{2} \ni(x, y) \longmapsto \int v d\left(\mu_{x}^{\omega_{1}} \otimes \mu_{y}^{\omega_{2}}\right)
$$

is the greatest multiply harmonic minorant of $v$ on $\omega_{1} \times \omega_{2}$. 
Lemma 2.3 Let $\omega_{1}$ and $\omega_{2}$ be two relatively compact domains in $\Omega_{1}$ and $\Omega_{2}$, respectively. If $u$ is a quasi multiply superharmonic function on $\Omega_{1} \times \Omega_{2}$, then for $(x, y) \in \omega_{1} \times \omega_{2}$ and $j=1,2$, we have

$$
\iint u d \mu_{x}^{\omega_{1}} d \mu_{y}^{\omega_{2}}=\iint \hat{u} d \mu_{x}^{\omega_{1}} d \mu_{y}^{\omega_{2}}=\iint \hat{u}^{j} d \mu_{x}^{\omega_{1}} d \mu_{y}^{\omega_{2}} .
$$

Proof Since $u$ is locally lower bounded, there exists a number $A$ such that $u+A>0$. So, without loss of generality we may assume that $u$ is positive. Also, we may assume that the function $u$ is bounded above, for otherwise we could consider $u_{n}=\min \{u, n\}$ and then take the limit.

Since $u$ is quasi multiply superharmonic, therefore for every $(x, y) \in \omega_{1} \times \omega_{2}$ we have $u(x, y) \geq \iint u d \mu_{x}^{\omega_{1}} d \mu_{y}^{\omega_{2}}$. The function $(x, y) \longmapsto \iint u d \mu_{x}^{\omega_{1}} d \mu_{y}^{\omega_{2}}$ is continuous in $\omega_{1} \times \omega_{2}$ and $u \geq \hat{u}$. Therefore,

$$
\hat{u}(x, y) \geq \iint u d \mu_{x}^{\omega_{1}} d \mu_{y}^{\omega_{2}} \geq \iint \hat{u} d \mu_{x}^{\omega_{1}} d \mu_{y}^{\omega_{2}} \forall(x, y) \in \omega_{1} \times \omega_{2} .
$$

Since the function $(x, y) \rightarrow \iint u d \mu_{x}^{\omega_{1}} d \mu_{y}^{\omega_{2}}$ is multiply harmonic, the above lemma implies that

$$
\iint u d \mu_{x}^{\omega_{1}} d \mu_{y}^{\omega_{2}}=\iint \hat{u} d \mu_{x}^{\omega_{1}} d \mu_{y}^{\omega_{2}}
$$

The other equalities follow because $\hat{u} \leq \hat{u}^{i} \leq u$ for $i=1,2$.

Lemma 2.4 Let $\sigma$ be a relatively compact domain in $\Omega_{2}$. Let $w$ be a quasi multiply superharmonic function on $\Omega_{1} \times \sigma$ such that the function

$$
\sigma \ni y \longmapsto w(x, y)
$$

is harmonic in $\sigma$ for each $x \in \Omega_{1}$. Then,

(i) $\hat{w}=\hat{w}^{1}$.

(ii) $\hat{w}=w$ everywhere except possibly on a set of the form $P \times \sigma$, where $P$ is a polar set in $\Omega_{1}$.

Proof (i) Let $(x, y) \in \Omega_{1} \times \sigma$. Let $\omega_{1}^{n}$ be a sequence of regular domains in $\Omega_{1}$ such that $\bigcap_{n} \omega_{1}^{n}=\{x\}$, and let $\omega_{2}^{m}$ be a sequence of regular domains in $\sigma$ such that $\bigcap_{n} \omega_{2}^{n}=\{y\}$. Since $t \longmapsto w(s, t)$ is harmonic in $\sigma$ for each $s \in \Omega_{1}$, for each positive integer $n$, we have

$$
\int w(s, t) d \mu_{y}^{\omega_{2}^{n}}(t)=w(s, y) \forall s \in \Omega_{1} .
$$

By integrating both sides with respect to $\mu_{x}^{\omega_{1}^{n}}$, we get

$$
\iint w(s, t) d \mu_{x}^{\omega_{1}^{n}}(s) d \mu_{y}^{\omega_{2}^{n}}(t)=\iint w(s, t) d \mu_{y}^{\omega_{2}^{n}}(t) d \mu_{x}^{\omega_{1}^{n}}(s)=\int w(s, y) d \mu_{x}^{\omega_{1}^{n}}(s) .
$$

Thus,

$$
\hat{w}(x, y)=\sup _{n} \iint w d \mu_{x}^{\omega_{1}^{n}} d \mu_{y}^{\omega_{2}^{n}}=\sup _{n} \int w(., y) d \rho_{x}^{\omega_{1}^{n}}=\hat{w}^{1}(x, y) .
$$

(ii) Fix $\tilde{y} \in \sigma$, and let $P=\left\{x \in \Omega_{1}: \hat{w}^{1}(x, \tilde{y})<w(x, \tilde{y})\right\}$. Since $w(., \tilde{y})$ is a quasi superharmonic function in $\Omega_{1}$, Theorem 2.1 implies that the set $P$ is a polar set in $\Omega_{1}$ and $w(x, \tilde{y})=\hat{w}^{1}(x, \tilde{y}) \forall x \in \Omega_{1} \backslash P$. Let

$$
E=\left\{(x, y) \in \Omega_{1} \times \sigma: \hat{w}^{1}(x, y)<w(x, y)\right\} .
$$


Now, we show that $E=P \times \sigma$. Let $\left(x_{1}, y_{1}\right) \in P \times \sigma$. Since $x_{1} \in P$, we have $w\left(x_{1}, \tilde{y}\right)>$ $\hat{w}^{1}\left(x_{1}, \tilde{y}\right)$. Let $h(y)=w\left(x_{1}, y\right)-\hat{w}^{1}\left(x_{1}, y\right)$ for $y \in \sigma$. It is clear that the function $h \geq 0$ and $h(\tilde{y})>0$. Moreover, $h$ is a superharmonic function in $\sigma$; in fact, $y \mapsto w\left(x_{1}, y\right)$ is harmonic by the assumption. Using Eq. (4), the function $y \mapsto \hat{w}^{1}\left(x_{1}, y\right)$ can be written as the supremum of a nondecreasing sequence of harmonic functions, so it is harmonic by Harnack's convergence theorem.

Therefore, $h(y)>0 \forall y \in \sigma$, in particular $h\left(y_{1}\right)>0$. Hence, $w\left(x_{1}, y_{1}\right)>\hat{w}^{1}\left(x_{1}, y_{1}\right)$, which implies that $\left(x_{1}, y_{1}\right) \in E$. Because $\left(x_{1}, y_{1}\right)$ is arbitrary in $P \times \sigma, P \times \sigma \subseteq E$. On the other hand, if $\left(x_{1}, y_{1}\right) \in E$, then $w\left(x_{1}, y_{1}\right)>\hat{w}^{1}\left(x_{1}, y_{1}\right)$. Let $g(y)=w\left(x_{1}, y\right)-\hat{w}^{1}\left(x_{1}, y\right)$ for $y \in \sigma$. With the same argument as above, $g$ is a nonnegative superharmonic function on $\sigma$ with $g\left(y_{1}\right)>0$. So, $g(y)>0 \forall y \in \sigma$, and in particular $g(\widetilde{y})>0$. Therefore, $w\left(x_{1}, \widetilde{y}\right)>\hat{w}^{1}\left(x_{1}, \tilde{y}\right)$, and hence, $x_{1} \in P$ and $\left(x_{1}, y_{1}\right) \in P \times \sigma$. As $\left(x_{1}, y_{1}\right)$ arbitrary, we conclude that $E \subset P \times \sigma$.

Lemma 2.5 Let $u$ be a quasi multiply superharmonic function on $\Omega_{1} \times \Omega_{2}$, and let $\sigma$ be a regular domain in $\Omega_{2}$. Define $w$ on $\Omega_{1} \times \sigma$ such that

$$
w:(x, y) \longmapsto \int u(x, .) d \mu_{y}^{\sigma} .
$$

Then,

(i) $\hat{w}=\hat{w}^{1}$.

(ii) There exists a polar set $P$ in $\Omega_{1}$ such that $\hat{w}(x, y)=w(x, y) \forall(x, y) \notin P \times \sigma$.

(iii) $\hat{w}(x, y)=\int \hat{u}^{1}(x,.) d \mu_{y}^{\sigma}, \forall(x, y) \in \Omega_{1} \times \sigma$.

(iv) If $E=\left\{(x, y) \in \Omega_{1} \times \sigma: \int \hat{u}^{1}(x,.) d \mu_{y}^{\sigma}<\int u(x,.) d \mu_{y}^{\sigma}\right\}$, then $E=P \times \sigma$ for some polar set $P$ in $\Omega_{1}$.

Proof (i) and (ii) follow directly from Lemma 2.4 because the function $w$ satisfies the assumptions of the lemma.

(iii) Let $(x, y) \in \Omega_{1} \times \sigma$. Let $\omega_{n}$ be a sequence of regular domains in $\Omega_{1}$ such that $\bigcap_{n} \omega_{n}=\{x\}$. Now,

$$
\begin{array}{rlr}
\hat{w}(x, y)=\hat{w}^{1}(x, y) & =\sup _{n} \int w(., y) d \mu_{x}^{\omega_{n}} & \\
& =\sup _{n} \iint u d \mu_{y}^{\sigma} d \mu_{x}^{\omega_{n}} & \text { (by definition of } w) \\
& =\sup _{n} \iint u d \mu_{x}^{\omega_{n}} d \mu_{y}^{\sigma} & \text { (by Fubini's theorem ) } \\
& =\sup _{n} \iint \hat{u}^{1} d \mu_{x}^{\omega_{n}} d \mu_{y}^{\sigma} & \text { (by Eq. (8)) } \\
& =\int \sup _{n}\left(\int \hat{u}^{1} d \mu_{x}^{\omega_{n}}\right) d \mu_{y}^{\sigma} \quad \text { (Monotone convergence theorem) } \\
& =\int \hat{u}^{1}(x, .) d \mu_{y}^{\sigma} . &
\end{array}
$$

(iv) Follows directly from the first three parts.

Now, we state and prove our main result.

Theorem 2.4 Let $u$ be a Borel function defined on a product of two Euclidean domains $\Omega_{1} \times$ $\Omega_{2}$, such that $u$ is locally lower bounded. Let $\hat{u}$ be the lower semicontinuous regularization of $u$. Then, $u$ is quasi multiply superharmonic function if and only if 
(i) $\hat{u}$ is multiply superharmonic on $\Omega_{1} \times \Omega_{2}$, and

(ii) $u=\hat{u}$ everywhere except in a negligible set.

Proof $(\Leftarrow)$ Suppose (i) and (ii). To show that $u$ is quasi multiply superharmonic, we need to show that $u$ satisfies inequality (6).

Let $(x, y) \in \Omega_{1} \times \Omega_{2}$. Let $\omega_{1}, \omega_{2}$ be relatively compact open sets such that $\omega_{1} \times \omega_{2} \subset$ $\Omega_{1} \times \Omega_{2}$. If $x \notin \omega_{1}$ or $y \notin \omega_{2}$, inequality (6) obviously holds. Suppose $(x . y) \in \omega_{1} \times \omega_{2}$. The set $\{\hat{u}<u\}$ is a $\mu_{x}^{\omega_{1}} \otimes \mu_{y}^{\omega_{2}}$-measurable negligible set; therefore, by Fubini's Theorem $\left(\mu_{x}^{\omega_{1}} \otimes \mu_{y}^{\omega_{2}}\right)(\{\hat{u}<u\})=0$. Now,

$$
\begin{aligned}
u(x, y) \geq \hat{u}(x, y) & \geq \int \hat{u} d\left(\mu_{x}^{\omega_{1}} \otimes \mu_{y}^{\omega_{2}}\right) \\
& =\int_{\{\hat{u}<u\}} \hat{u} d\left(\mu_{x}^{\omega_{1}} \otimes \mu_{y}^{\omega_{2}}\right)+\int_{\{\hat{u}=u\}} \hat{u} d\left(\mu_{x}^{\omega_{1}} \otimes \mu_{y}^{\omega_{2}}\right) \\
& =\int u d\left(\mu_{x}^{\omega_{1}} \otimes \mu_{y}^{\omega_{2}}\right) .
\end{aligned}
$$

This shows that $u$ is quasi multiply superharmonic function.

$(\Rightarrow)$ Suppose that $u$ is a quasi multiply superharmonic function.

Proof of (i): Theorem 2.3.

Proof of (ii ): Let $B_{1}=\left\{\omega_{1}^{n}\right\}$ be a base of regular domains in $\Omega_{1}$, and let $B_{2}=\left\{\omega_{2}^{n}\right\}$ be a base of regular domains in $\Omega_{2}$. Let

$$
\begin{aligned}
& A_{1}=\bigcup_{n}\left\{(x, y) \in \Omega_{1} \times \omega_{2}^{n}: \int \hat{u}^{1}(x, .) d \mu_{y}^{\omega_{2}^{n}}<\int u(x, .) d \mu_{y}^{\omega_{2}^{n}}\right\}, \\
& A_{2}=\bigcup_{n}\left\{(x, y) \in \omega_{1}^{n} \times \Omega_{2}: \int \hat{u}^{2}(., y) d \mu_{x}^{\omega_{1}^{n}}<\int u(., y) d \mu_{x}^{\omega_{1}^{n}}\right\},
\end{aligned}
$$

and

$$
B=\left\{(x, y) \in \Omega_{1} \times \Omega_{2}: \hat{u}^{1}(x, y)<u(x, y) \text { and } \hat{u}^{2}(x, y)<u(x, y)\right\} .
$$

By Lemma 2.5, the sets $A_{1}, A_{2}$ are negligible sets. For each $x_{0} \in \Omega_{1}$, the section $B_{x_{0}}\left(\Omega_{2}\right)=\left\{y \in \Omega_{2}:\left(x_{0}, y\right) \in B\right\}$ is a subset of the set $\left\{y \in \Omega_{2}: \hat{u}^{2}\left(x_{0}, y\right)<u\left(x_{0}, y\right)\right\}$, which, by Theorem 2.1, is polar because the function $y \mapsto u\left(x_{0}, y\right)$ is a quasi superharmonic function on $\Omega_{2}$. So $B_{x_{0}}\left(\Omega_{2}\right)$ is polar. Similarly, for each $y_{0} \in \Omega_{2}$ the section $B_{y_{0}}\left(\Omega_{1}\right)$ is polar, and hence, the set $B$ is a negligible set. Therefore, the set $E=A_{1} \cup A_{2} \cup B$ is a negligible set. To complete the proof, we need to show that if $(x, y) \notin E$, then $\hat{u}(x, y)=u(x, y)$.

If $(x, y) \in\left(\Omega_{1} \times \Omega_{2}\right) \backslash E$, then $(x, y) \notin B$, which implies that either $\hat{u}^{1}(x, y)=u(x, y)$ or $\hat{u}^{2}(x, y)=u(x, y)$. Without loss of generality we may assume that $\hat{u}^{1}(x, y)=u(x, y)$. Let $\omega_{1}^{n}$ be a sequence in $B_{1}$ such that $\bigcap_{n} \omega_{1}^{n}=\{x\}$ and $\omega_{2}^{n}$ a sequence in $B_{2}$ such that $\bigcap_{n} \omega_{2}^{n}=\{y\}$. Now,

$$
\begin{aligned}
\hat{u}(x, y) & =\sup _{n} \sup _{m} \iint u d \mu_{x}^{\omega_{1}^{n}} d \mu_{y}^{\omega_{2}^{m}} \\
& =\sup _{n} \sup _{m} \int\left(\int u d \mu_{y}^{\omega_{2}^{m}}\right) d \mu_{x}^{\omega_{1}^{n}} \\
& =\sup _{n} \int\left(\sup _{m} \int u d \mu_{y}^{\omega_{2}^{m}}\right) d \mu_{x}^{\omega_{1}^{n}} \\
& =\sup _{n} \int \hat{u}^{2}(., y) d \mu_{x}^{\omega_{1}^{n}}
\end{aligned}
$$

(Fubini's theorem)

(Monotone convergence theorem) 


$$
\begin{array}{ll}
\left.=\sup _{n} \int u(., y)\right) d \mu_{x}^{\omega_{1}^{n}} & \text { (because } \left.(x, y) \notin A_{2}\right) \\
=\hat{u}^{1}(x, y)=u(x, y) . &
\end{array}
$$

Corollary 2.1 Let $u$ be a Borel function defined on a product of two Euclidean domains $\Omega_{1} \times \Omega_{2}$, such that $u$ is locally lower bounded. Suppose that for each $(x, y) \in \Omega_{1} \times \Omega_{2}, u$ satisfies the following inequality:

$$
u(x, y) \geq \int u d \mu \forall \mu \in J_{(x, y)}(M S) .
$$

Then, $\hat{u}$ is multiply superharmonic on $\Omega_{1} \times \Omega_{2}$ and $u=\hat{u}$ everywhere except in a negligible set.

Corollary 2.2 Let $\left\{u_{\alpha}\right\}$ be a family of uniformly locally lower bounded multiply superharmonic functions with $u=\inf u_{\alpha}$. Then, $\hat{u}$ is a multiply superharmonic function and $\hat{u}=u$ everywhere except on a negligible set.

Finally, we remark that Theorem 2.4 is generalization of the following result of Singman (see [12]).

Corollary 2.3 (Singman) Let $\left\{u_{n}\right\}$ be a decreasing sequence of uniformly locally lower bounded multiply superharmonic functions with limit function $u$. Then, $\hat{u}$ is a multiply superharmonic function and $\hat{u}=u$ everywhere except on a negligible set.

\section{References}

1. Alakhrass, M.: Jensen measures in product harmonic spaces. Annali di Matematica. doi:10.1007/ s10231-013-0388-9

2. Alakhrass, M., Hansen, W.: Infima of superharmonic functions. Ark. Mat. 50(2), 231-235 (2012)

3. Alakhrass, M.: Superharmonic and multiply superharmonic functions and Jensen measures in axiomatic Brelot spaces. Ph.D. thesis, McGill University, Montreal (2009)

4. Brelot, M.: Lectures on Potential Theory, Tata Institute, No. 19, Bombay 1960, re-issude (1967)

5. Cole, B.J., Ransford, T.J.: Subharmonicity without upper semicontinuity. J. Funct. Anal. 147, 420-442 (1997)

6. Cole, B.J., Ransford, T.J.: Jensen measures and harmonic measures. J. Reine Angew. Math. 541, 29-53 (2001)

7. Gowrisankaran, K.N.: Multiply harmonic functions. Nagoya Math. J. 28, 27-48 (1966)

8. Gowrisankaran, K.N.: Iterated fine limits and iterated nontangental limits. Trans. Am. Math. Soc. 173, 71-92 (1972)

9. Gowrisankaran, K.N.: Multiply superharmonic functions. Ann. Inst. Fourier 25, 235-244 (1975)

10. Gowrisankaran, K.N.: Negligible sets and good functions on polydisc. Ann. Inst. Fourier 29, 211-222 (1979)

11. Gowrisankaran, K.N.: Measurability of functions in product spaces. Am. Math. Soc. 31(2), 485-488 (1972)

12. Singman, D.: Exceptional sets in a product of harmonic spaces. Math. Ann. 262, 29-43 (1983) 